\title{
UN ESTUDIO EXPLORATORIO SOBRE EL DESARROLLO DE COMPETENCIAS DIGITALES EN LA EDUCACIÓN SUPERIOR
}

Exploratory Study Regarding Digital Competence Development in Higher Education

\section{Carolina España Chavarría}

Universidad Nacional, Costa Rica, cespanac@yahoo.com.mx

\author{
Xinia Corrales Escalante \\ Universidad Nacional, Costa Rica, xinia.corrales.escalante@una.cr
}

$$
\text { doi: } h \text { ttp://dx.doi.org/10.15359/abra.34-49.5 }
$$

Resumen: El siguiente es un estudio exploratorio para obtener un primer conocimiento sobre el desarrollo de competencias digitales alcanzadas por el grupo estudiantil participante del curso Elaboración Integral de Documentos III de las carreras Administración de Oficinas y Educación Comercial impartido en la Universidad Nacional de Costa Rica. El objetivo del estudio es conocer la opinión discente sobre la importancia otorgada al desarrollo de competencias digitales en el proceso de formación universitaria. Como principales resultados se evidencia una formación inicial relativamente insuficiente ante estas competencias, consideradas necesarias para un mejor desempeño en la sociedad contemporánea. Las consecuencias se asocian al escaso conocimiento, a la falta de habilidades y al desarrollo de actitudes del grupo estudiantil que dificultan su quehacer para afrontar los desafíos del mundo actual.

Palabras claves: Competencias digitales; formación universitaria; conocimiento; aprendizaje.

Abstract: This exploratory study was aimed at learning about 
the digital skills acquired by a group of students from the course entitled Comprehensive Preparation of Documents III for the Office Management and Commercial Education Programs at Universidad Nacional de Costa Rica. The objective was to obtain the opinions of students regarding the importance given to the development of digital skills in the university education process. As part of the principal results, there is evidence of relatively insufficient initial training in digital skills, which are considered necessary to better perform in contemporary society. The consequences are associated with the lack of knowledge and skills, as well as some students' attitudes that hinder the performance required to face the challenges of today's world.

Keywords: Digital skills; university education; knowledge; learning.

Fecha de recepción: 03-05-2014 Fechas de reenvíos: 17-07-14/01-08-14/ 16-09-14/30-09-14/02-1014 Fecha de aceptación: 02-10-2014 Fecha de publicación: 02-12-2014

El estudio de los nuevos fenómenos sociales y su vinculación con los avances de la información y el conocimiento están caracterizados, entre otros asuntos, por los procesos tecnológicos (Brunner, 2003; Castells. 2005; Tedesco, 1999) en donde las Tecnologías de la Información y Comunicación (TIC) y su capacidad de conexión obligan el rediseño de los contextos de formación.

El logro de un mejoramiento de la educación como consecuencia del cambiante contexto socio histórico, está vinculado con las acciones pedagógicas impulsadas por el grupo docente y las posibilidades para desarrollar conocimiento, habilidades y valores que aproveche el grupo estudiantil.

Actualmente, los contextos aprendientes están llamados a posibilitar espacios para la construcción del conocimiento a partir del desarrollo de diversas competencias genéricas y específicas, en donde las de orden digital son de gran importancia pues facultan al grupo estudiantil para que pueda buscar, producir, tratar, difundir y transformar la información para el beneficio de su desarrollo integral. Lo anterior, nos hace creer que las competencias digitales demandadas por la 
sociedad actual a quienes se encuentran vinculados con los procesos de formación universitaria, se asocian no solo con las habilidades, los conocimientos y las actitudes con respecto a las tecnologías, sino a su pertinente y funcional aplicación frente a una meta concreta.

De allí la necesidad de emprender estudios como este con el fin de dar a conocer el valor otorgado al desarrollo de competencias digitales en el proceso de formación universitaria vivenciado por el grupo participante del curso Elaboración Integral de Documentos III de las carreras Administración de Oficinas y Educación Comercial impartido en la Escuela de Secretariado Profesional de la Universidad Nacional de Costa Rica, contexto del presente análisis. Lo anterior como consecuencia de la interrogante que da pie a la indagación realizada: ¿la formación universitaria promueve el desarrollo de competencias digitales en el estudiantado?

Lo expuesto condujo al objetivo general de este estudio, el cual fue diagnosticar el desarrollo de competencias digitales alcanzadas por el grupo estudiantil meta. Entre los objetivos específicos están: identificar el valor otorgado al desarrollo de competencias digitales en la formación universitaria, analizar la congruencia entre las competencias digitales que demanda la sociedad y las que promueve la enseñanza universitaria y determinar las competencias digitales necesarias en el aprendizaje y desarrollo profesional del grupo estudiantil. Lo anterior, con el fin de determinar la funcionalidad y permanencia de los aprendizajes promovidos, así como su incidencia en la construcción del perfil profesional requerido para enfrentar los retos que la sociedad contemporánea demanda.

\section{Aproximación teórica}

El desarrollo de competencias para la vida como parte de los procesos de transformación educativa que provoca la modernidad, supone ser parte de las funciones planeadas, deseadas sistematizadas y evaluadas que complementan el hacer pedagógico. Este proceso debe generar motivación y significancia en el alumnado, además de posibilitar el desarrollo de las competencias digitales básicas que le permitirán desenvolverse en una sociedad tecnológicamente activa. 
En la actualidad y de acuerdo con Castells (2009), la condición de "nativos digitales" de los jóvenes les confiere una posición dominante frente a sus mayores. Su uso cotidiano de los medios digitales de información y comunicación les ha permitido desarrollar nuevas formas de relación y construir sus propios espacios de autonomía colectiva. Las instituciones educativas deben afrontar el reto de adecuarse a la realidad de sus públicos para frenar ese desfase cultural-tecnológico que es un hecho. Sobre esta realidad, el profesorado universitario debe estar preparado para promover en el estudiantado las competencias necesarias para que este grupo se desenvuelva en un mundo globalizado y digital. En esa misma línea de ideas, Canales y España (2013), manifiestan que la contribución y repercusión de las nuevas tecnologías de la información (TIC) que se llevan a cabo en los entornos aprendientes incide significativamente en el tipo de relaciones humanas desarrolladas. La forma de comunicarse, de entender y procesar lo que sucede está impactada por los entornos y recursos tecnológicos existentes, esto como consecuencia de su potencial social y educativo. Por lo tanto, la influencia de las TIC deberá ser considerada como un instrumento más con que cuenta el proceso de aprendizaje para alcanzar su fin formativo, construir conocimiento y potenciar el entusiasmo por la investigación y la innovación.

Dentro de este contexto y como respuesta a la sociedad del conocimiento la Universidad Nacional (UNA) definió en su modelo pedagógico (UNA Gaceta, 2007), el uso de las TIC como un medio para la promoción de los aprendizajes y el mejoramiento de los procesos educativos. Desde esa perspectiva, las tecnologías en los procesos de enseñanza y aprendizaje se conciben como un medio que colabora en la promoción del aprendizaje y en la calidad académica. Las tecnologías permiten la creación de diversos escenarios de aprendizaje, caracterizados por ser flexibles, diversos, interactivos, colaborativos, y ampliados en conexiones, en donde académicos y estudiantes se comunican, comparten, dialogan, aprenden juntos y ambos construyen conocimiento para superar las barreras de espacio y tiempo. En estos ambientes para el aprendizaje las tecnologías facilitan la interacción entre profesores, contenidos, estudiantes y otros expertos, para una formación integral. Es así, como los usos de las tecnologías en el acto 
educativo se conciben en la UNA como colaboradoras en la formación de un estudiante crítico, flexible y comprometido con la sociedad.

Ahora bien, ¿qué podemos entender por competencias digitales?, son múltiples las definiciones pero de acuerdo con el documento titulado "Competencias clave para el aprendizaje permanente", publicado por el Parlamento Europeo en el año 2006, la competencia digital exige una buena comprensión y amplios conocimientos sobre la naturaleza, la función y las oportunidades de las Tecnologías para la Información y Comunicación (TIC) en situaciones cotidianas de la vida privada, social y profesional, lo principal de la competencia digital es que debe posibilitar el uso seguro y crítico de las TIC para el trabajo, el ocio y la comunicación. Por consiguiente, se sustenta en las competencias básicas en materia de TIC, es decir el uso de computadoras y otros dispositivos para obtener, evaluar, almacenar, producir, presentar e intercambiar información, comunicarse y participar en redes de colaboración a través de internet.

Consciente de la necesidad de formar en competencias digitales, la UNA debe replantearse los procesos educativos que se gestan en las diferentes facultades, centros y sedes, además de poner atención al desarrollo de competencias esenciales para la vida, porque según el Área (2010) las universidades deben ofrecer a la ciudadanía una educación superior, donde, entre otras metas, al estudiantado se forme como sujeto competente para afrontar los complejos desafíos de la cultura, el conocimiento, la ciencia, la economía y de las interacciones en este siglo XXI.

\section{Metodología}

Como se indicó al inicio de este trabajo, lo que se busca es contribuir al mejoramiento de los procesos de formación universitaria para fomentar el desarrollo de competencias digitales en el individuo como la vía que facilita la construcción de su conocimiento. Por tanto, el esquema de presentación y exposición de resultados fue construido a partir de un desarrollo conceptual actualizado de las relaciones que se establecen entre las percepciones del estudiantado y lo que acontece en el seno de la práctica formativa. 
A continuación se presenta el proceso seguido para el desarrollo de la investigación. Su planteamiento se divide en dos grandes segmentos. El primero, dirigido a conocer el objeto de la investigación en concordancia con los objetivos trazados y el universo de estudio (contexto y actores). El segundo, orientado a detallar el método de exploración, las técnicas e instrumentos utilizados, las actitudes y consideraciones de la población meta, algunos detalles significativos del contexto de estudio, así como otros datos fundamentales que permitieron emprender el análisis requerido para esta investigación.

\section{El objeto estudio y el escenario de la investigación}

El objeto de este estudio es la relación existente entre los procesos de formación universitaria y el desarrollo de competencias digitales en el alumnado. También, y con el propósito de profundizar en el análisis, se consideró como contexto la Facultad de Ciencias Sociales de la Universidad Nacional de Costa Rica (FCS), recinto de formación del colectivo discente consultado y nicho laboral del equipo investigador, estas últimas quienes se han dado a la tarea de explorar en profundidad lo que subyace en el contexto de aprendizaje promovido en el curso Elaboración Integral de Documentos III de las carreras Administración de Oficinas y Educación Comercial de la Escuela de Secretariado Profesional.

El contexto para el estudio se escogió de forma intencional puesto que la FCS no solo es diversa en sus disciplinas, sino que también se dedica casi en su totalidad a la docencia (solo dos de las diez unidades académicas son centros de investigación.

Producto de las características del contexto anteriormente citadas y por el interés de acercar la realidad educativa que se vive en la FCS, se recurrió al paradigma de investigación naturalista, porque la finalidad del estudio es comprender e interpretar la realidad, los significados de las personas, las percepciones, las interacciones y las acciones (Barrantes, 2013) con un enfoque cualitativo para explorar y describir la situación objeto de este estudio que de acuerdo con autores como Goetz y Lecompte (1988) establecen que es parte de los caminos que permiten desarrollar en el marco de la investigación 
social, un conocimiento comprometido con la verdad y con el bienestar de los seres humanos, de manera que, aparte de compromiso entre las personas, surja una interacción y una negociación constante.

Es importante aclarar que solo para la categoría las TIC en la formación universitaria se aplicó un análisis cuantitativo descriptivo porque era necesario medir el acceso y uso de las TIC por parte del estudiantado en la formación universitaria y mostrar la realidad mediante números.

De esa manera se caracteriza la metodología utilizada en esta investigación, pues el interés que condujo este acto investigativo fue el abordaje complejo, pero el necesario análisis al que debe someterse la calidad de la formación promovida y la significancia otorgada por los actores principales del proceso (el colectivo estudiantil), a las competencias digitales desarrolladas en el proceso formativo universitario y la pertinencia que tiene el mismo para el desarrollo de sus vidas personales y profesionales.

\section{Métodos de selección de participantes, de exploración y análisis}

Para realizar este análisis, se consideró dos grupos de estudiantes del Curso Elaboración Integral de Documentos III, del IV nivel de Bachillerato en Administración de Oficinas y Educación Comercial, alrededor de un 90\% (36 estudiantes) de género femenino y con edades entre un rango de 19 a 29 años. Se tomó en cuenta esta población para conocer la opinión y experiencia, ya que están por concluir su bachillerato y algunos laboran, por lo que se puede identificar si las competencias digitales en la formación universitaria son importantes o no para ellas/os y si tienen pertinencia para el desarrollo de sus vidas personales y profesionales.

Por otra parte y si bien es cierto en la investigación cualitativa tal y como lo señala, Hernández, Fernández y Baptista (2010) el investigador cualitativo utiliza técnicas para recolectar datos, como la observación no estructurada, entrevistas abiertas, revisión de documentos, discusión en grupo, evaluación de experiencias personales, registro de historias de vida, e interacción e introspección con grupos o comunidades, en este estudio se escogió el cuestionario estructurado con preguntas abiertas y cerradas como medio de recolección de información (Thomas y Nelson, 
2007). Lo que motivó la escogencia fue la necesidad de obtener por medio de la formulación de preguntas específicas, las respuestas que permitieran profundizar en la percepción del estudiantado participante y así alcanzar el objetivo de la investigación. Además, el cuestionario es un instrumento claro, ordenado, amigable y conocido por el grupo de estudiantes, el cual fue validado por las especialistas en la inmersión que hicieron en el trabajo de campo. Asimismo, se determinó que la construcción y el contenido del instrumento se ajustan al objetivo del proceso de investigación planteado. El cuestionario contó con tres dimensiones a saber: las TIC en la formación universitaria, en esta dimensión se efectuó una simple medición numérica para obtener datos sólidos que permitan fundamentar el acceso a las TIC que tiene la población investigada, la segunda dimensión fue procesos de aprendizaje e innovación y la tercera competencias digitales.

Para la obtención de la información se contempló una población total de treinta y seis estudiantes de los 41 matriculados en los dos grupos del curso Elaboración Integral de Documentos III ofertado en el II ciclo del 2013. En este período las investigadoras, una de ellas docente a cargo de los grupos, realizaron una aplicación autoadministrada de los cuestionarios en el aula física y en el aula virtual. Una vez recopilados los instrumentos se procedió a codificar los datos de forma manual y fueron procesados mediante el diseño de gráficos con la herramienta Microsoft Word. Cada subtema se analizó y tabuló por separado para garantizar un debido ordenamiento de la información y con ello dar una mejor lectura de los datos que arrojaba la técnica aplicada para el desarrollo del trabajo de campo. Para la segunda y tercera dimensión se identificaron las diferentes percepciones del grupo de estudiantes, se ordenaron y agruparon en subcategorías tales como: recursos tecnológicos con que cuenta el estudiantado, acceso a Internet y tipo de conexión, cursos más innovadores, sistemas operativos y navegadores que utiliza, aprendizajes más importantes para ejercer su profesión, conocimientos que quedaron por fuera, cambios para asegurar la calidad de los cursos, y competencias digitales que debe poseer el estudiantado al finalizar la carrera. Posteriormente, las respuestas se narraron con el mismo lenguaje utilizado por los participantes, se relacionó con la teoría, los datos encontrados se interpretaron y analizaron por categorías 


\section{Hallazgos}

\section{Las TIC en la formación universitaria}

La incorporación de las Tecnologías para la Información y Comunicación en la vida del estudiantado universitario es una realidad, existen múltiples recursos tecnológicos como la televisión, la radio, el teléfono móvil, la computadora, las tabletas, el video, el Internet, Skype y las redes sociales que el grupo utiliza para comunicarse, socializar, acceder a información, interactuar y aprender, entre otras acciones, bajo esta afirmación, se les preguntó al grupo de estudiantes ¿con qué recursos tecnológicos cuenta? para saber en qué medida están familiarizados con el uso de las TIC, lo cual muestra que la totalidad tiene acceso a una computadora de escritorio personal (28\%) o a una computadora portátil (30\%). Además, un $40 \%$ de las personas encuestadas tiene acceso a un teléfono móvil. Si bien el acceso y uso de dispositivos móviles se propaga en la sociedad costarricense, se deriva de este estudio que el recurso tecnológico con menor acceso son las tabletas ya que del total del estudiantado solamente una persona cuenta con este dispositivo, como se presenta en figura 1. $1 \% 1 \%$

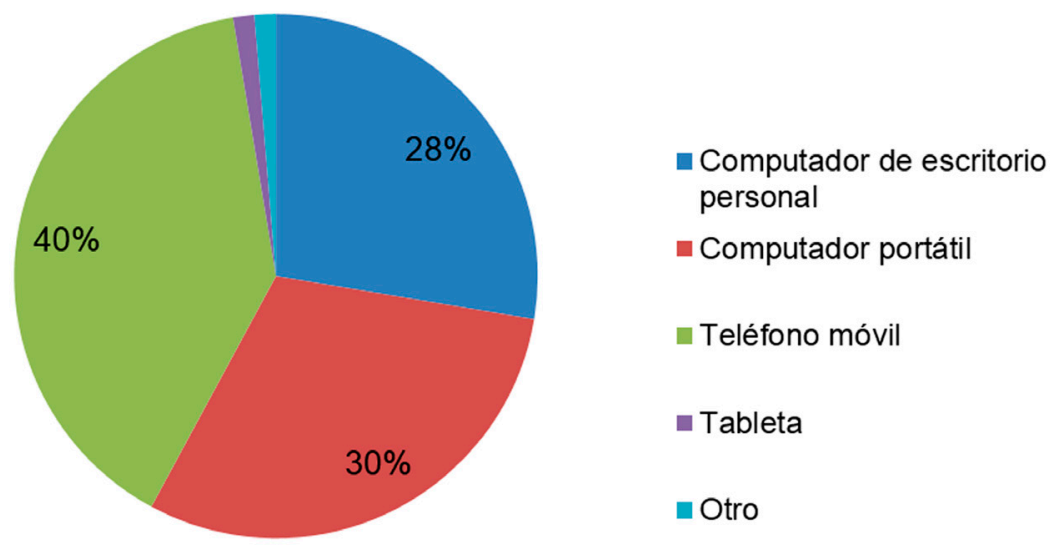

Figura 1. ¿Con qué recursos tecnológicos cuenta usted? Recursos tecnológicos. Fuente: elaboración propia, 2013 
Adicionalmente, en los figuras 2 y 3 se muestra el tipo de conexión que usan las personas encuestadas para acceder a Internet y los sistemas operativos más utilizados, de tal manera que se nota como un $36 \%$ del estudiantado encuestado (del total de 36 personas) se conectan en su mayoría a redes inalámbricas WIFI a través de dispositivos móviles y respecto al tipo de sistemas operativos que utilizan, $72 \%$ usan el sistema operativo Windows. Lo anterior refleja la necesidad de garantizar y mantener una plataforma estable de red inalámbrica institucional para el acceso a los servicios de Internet en general, el correo electrónico, el acceso a los sistemas de información universitarios, el acceso a bases de datos locales y externas, entre otros servicios. Además, debido a que esta población en su mayoría usa el sistema operativo Windows es importante promover en el estudiantado universitario otros softwares libres como Linux, que no requieren licencias de pago, y así reducir costos de operación y promover el uso de sistemas operativos menos susceptibles a ataques informáticos.

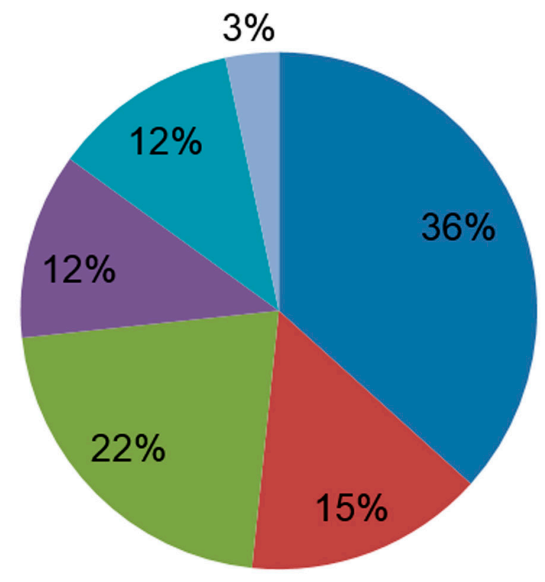

- Dispositivos portátiles conectados a redes Inalámbricas

- Red telefónica fija de alta velocidad

- Telefonía celular

- Red telefónica fija

Figura 2. ¿Qué tipo de conexiones utiliza para acceder a Internet? Tipos de conexiones. Fuente: elaboración propia, 2013 


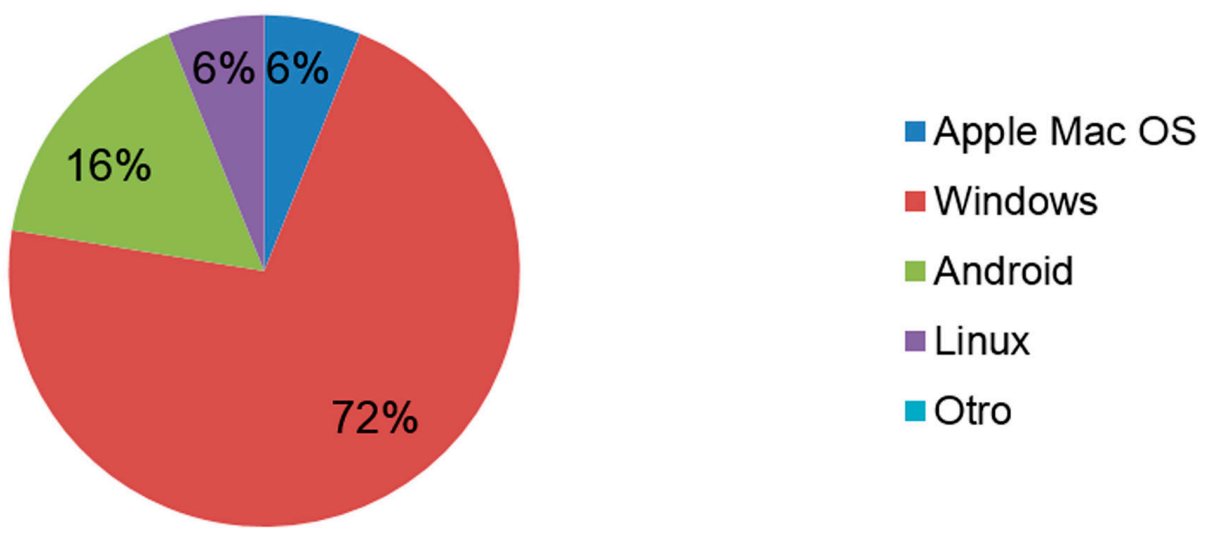

Figura 3. ¿Qué tipo de sistemas operativos utiliza? Sistemas operativos. Fuente: elaboración propia, 2013

De igual forma, el uso de Internet en los procesos educativos es cada vez mayor, y el estudiantado para acceder a todos los recursos e información utiliza los exploradores o navegadores web. Como resultado de la encuesta encontramos que el grupo de estudiantes utiliza como navegador preferido el Google Crome, pero se destaca el creciente uso de Fire Fox e Internet Explorer como navegadores alternativos y con mayor rendimiento (Figura 4).

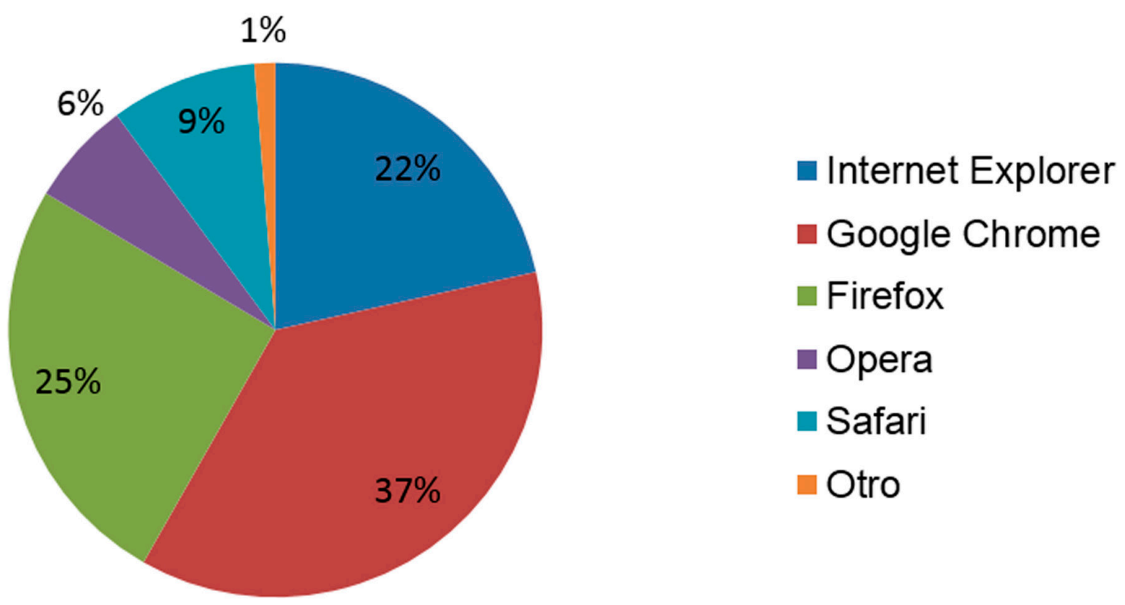

Figura 4. ¿Cuáles navegadores de Internet utiliza? Navegadores de Internet. Fuente: elaboración propia, 2013 
Al analizar esta subcategoría es posible concluir que la mayoría de los participantes tienen acceso a una computadora, a conexión a internet y utilizan diferentes programas para navegar y acceder a los servicios y medios de Internet en general. De ahí la importancia que los procesos educativos que se promueven en la universidad no pueden quedar al margen de esta realidad tecnológica y su incidencia supone un cambio significativo en el rol del estudiantado y docente con nuevas funciones, metodologías y motivaciones ante el aprendizaje.

\section{Procesos de aprendizaje e innovación}

En el modelo pedagógico de la Universidad Nacional se entienden los procesos de enseñanza y el aprendizaje como procesos sociales, históricos y culturales que van más allá de la mera transmisión del conocimiento (Universidad Nacional, 2007). Se fundamenta en el análisis y problematización de la realidad, del trabajo práctico e investigativo sobre el contexto en que se desenvuelve el estudiante y su carrera, en el desarrollo de competencias para la innovación y la resolución de problemas, la negociación de conflictos, el trabajo en equipo interdisciplinario, y la toma de decisiones con base en información confiable y oportuna. En consecuencia se les consultó al grupo de estudiantes, que según lo aprendido en los cursos de aplicación tecnológica ¿cuáles son los aprendizajes más importantes para ejercer su profesión?, en donde se afirmó y destacó lo siguiente:

Los siguientes argumentos se complementan con la pregunta ¿cuáles son los aprendizajes que se dejaron por fuera y son para usted necesarios a la hora de ejercer su profesión? En la tabla 2 se observa un resumen. 
Tabla. 1.

Aprendizajes más importantes para ejercer su profesión

\section{Usos}

1. Uso de los programas informáticos como: procesadores de textos, hojas de cálculo y programas para hacer presentaciones.

2. Aplicación de fórmulas y gráficos en Microsoft Excel

3. Diseño e implementación de base de datos.

4. Creación de sitios web.

5. Uso de Internet, sus servicios y medios.

6. Manipulación adecuada de herramientas tecnológicas para la oficina y sus actualizaciones.

7. Uso de exploradores y navegadores en Internet

8. Manejar información y compartirla por medio de programas en línea como el Dropbox.

9. Redactar informes y como darle formato a cada documento creado en una oficina.

Fuente: elaboración propia, 2013

Tabla 2.

Aprendizajes que se dejaron por fuera y son necesarios para ejercer su profesión

\section{Argumentos}

1. Uso de agendas electrónicas.

2. Conocimientos avanzados de los programas para hacer presentaciones Microsoft Power Point y Prezi.

3. El tema de páginas web debería de trabajarse de forma más amplia.

4. Aprender a descargar documentos, películas, sonidos, música y programas. 
Continuación tabla 2.

5. Conocer acerca de los macros especialmente en Excel.

6 . Se dejó por fuera tomar en cuenta los idiomas como inglés y portugués, la parte técnica que es esencial para poder competir en el mercado a la hora de aplicar para un puesto en especial.

7. Descargar música de internet.

8. Actualizar los programas de la computadora, el software, profundizar en más funciones específicas y especiales de cada programa con respecto a los cursos de contabilidad, comercio internacional, entre otros.

9. El uso de Publisher, Project y Autocad.

10. Sistemas para contabilidad, presupuestos y SPSS, que se usan mucho en la oficina.

11. Con respecto a los cursos que se han impartido sobre tecnología se dejó por fuera el utilizar el Outlook debido a que el laboratorio no contaba con el paquete adecuado para trabajarlo.

12. Profundizar más en la utilización del internet como herramienta de búsqueda efectiva para investigaciones.

13. En mi opinión un estudiante de Administración de Oficinas debe salir de la carrera manejando por completo los programas de Word, Excel, Access también debe ser capaz de realizar una investigación utilizando de manera adecuada la tecnología del internet y es muy importante también que el nivel de inglés sea excelente, es necesario que se mejore el nivel de inglés para poder asegurar que un estudiante graduado de Administración de Oficinas sea capaz de manejar esta lengua a nivel oral y escrito, de no ser así, no tiene sentido cursar ingles durante los cuatro años de carrera.

14. Organización de una videoconferencias y firma digital

Fuente: Elaboración propia, 2013

Como puede verse en las tablas 1 y 2 el grupo de estudiantes encuestados opinan acerca de la importancia de un conocimiento amplio y profundo de programas computacionales y herramientas tecnológicas para procesar información y datos, producir documentos, hacer cálculos, llevar estadísticas, organizar y mantener base de datos, uso de internet, sus servicios y medios, nivel de inglés, conocimientos prácticos y necesarios para ejercer su profesión con un nivel tecnológico avanzado, que les permita trabajar y resolver 
problemas con criticidad y creatividad para desempeñarse en cualquier área de una organización. De los resultados de la encuesta también se desprenden dos respuestas sobresalientes acerca de los contenidos que se quedaron por fuera, y que afirman lo siguiente:

“... Siento que no es tanto los contenidos que se quedaron por fuera, sino que el curso o la persona encargada de impartirlo esté actualizada con los programas de los cursos y tenga el manejo necesario de todos los contenidos para que los estudiantes no sientan que les faltó aprendizaje",

“... Dentro de los cursos de tecnología muchas veces se dejan aspectos y detalles por fuera, ya que muchos de los profesores que imparten el curso no tienen como carrera primaria informática, sino más bien son profesores egresados de la Escuela de Secretariado Profesional con conocimiento del manejo de los paquetes de Microsoft Office. Se debería capacitar más frecuentementeal profesoradoencargado de impartirestos cursos".

Estasopiniones invitana reflexionarsobrelaimportancia, responsabilidad y necesidad de la actualización y formación profesional permanente por parte del personal académico de la Escuela de Secretariado Profesional para generar procesos educativos innovadores, porque el rol que ejerce el grupo docentes es fundamental para la calidad y pertinencia de la educación universitaria y además como lo señala Fullan (1991): "Los cambios en educación dependen de lo que los profesores hacen y piensan, algo tan simple y tan complejo a la vez" (p.117).

Otra de las preguntas que se generaron en este estudio está relacionada con lo vivenciado por las personas encuestadas referente a su formación tecnológica universitaria, ¿cuáles cambios (profesorado, contenidos, metodología, evaluación de los procesos aprendizaje) haría usted para mejorar la calidad de los cursos universitarios ofertados por la Escuela de Secretariado Profesional y por qué? Para dar respuesta a esta interrogante el estudiantado ofrece algunas razones que son de interés y que se muestran en la figura 3. 


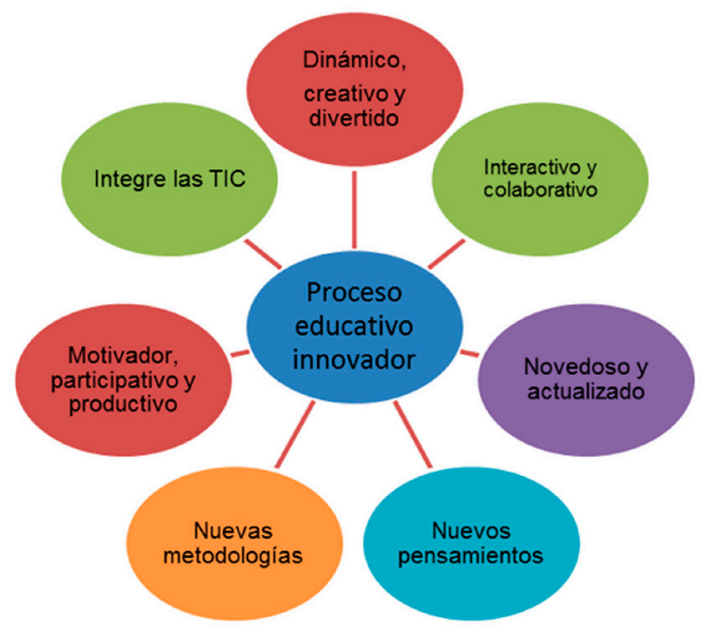

Figura 5. Cualidades más importantes de un proceso de formación innovador. Fuente: elaboración propia, 2013.

Sumado a la pregunta anterior se le consultó al estudiantado acerca de cuáles fueron los cursos más innovadores que recibió en su formación universitaria y que explique el porqué de su escogencia. En esta línea la población estudiantil expone aspectos relacionados con: “... son cursos que siempre enseñan algo nuevo y novedoso que puede ser aplicado en la carrera”, “... acceso a la información de forma innovadora”, “... obtuve el trabajo actual por solo haber llevado unas clases del curso Base de Datos", “... los cursos que más utilizo en mi vida”, “... Desde mi punto los cursos fueron y han sido muy innovadores porque se ha mezclado la parte teórica con la parte meramente laboral dentro del campo de administración de oficinas”, “... por la forma interactiva en que se imparten las lecciones con el apoyo de recursos como los videos", "... se preocupan por buscar siempre la innovación y retan al estudiante a crearla", "... el profesor realiza la clase de una manera muy novedosa, creativo, genera interés en los estudiantes, al mismo tiempo motiva al perfeccionamiento del idioma, lo vuelve productivo e interesante", "... la profe deja muchas tareas y trabajos y nos explica muchas cosas interesantes no es como otras profesoras que se limitan a enseñar lo básico", "... porque en ese curso lo aprendido y lo enseñado se puso en práctica y es mejor así”. Por lo anterior, los cursos que el estudiantado consideró más innovadores fueron: 


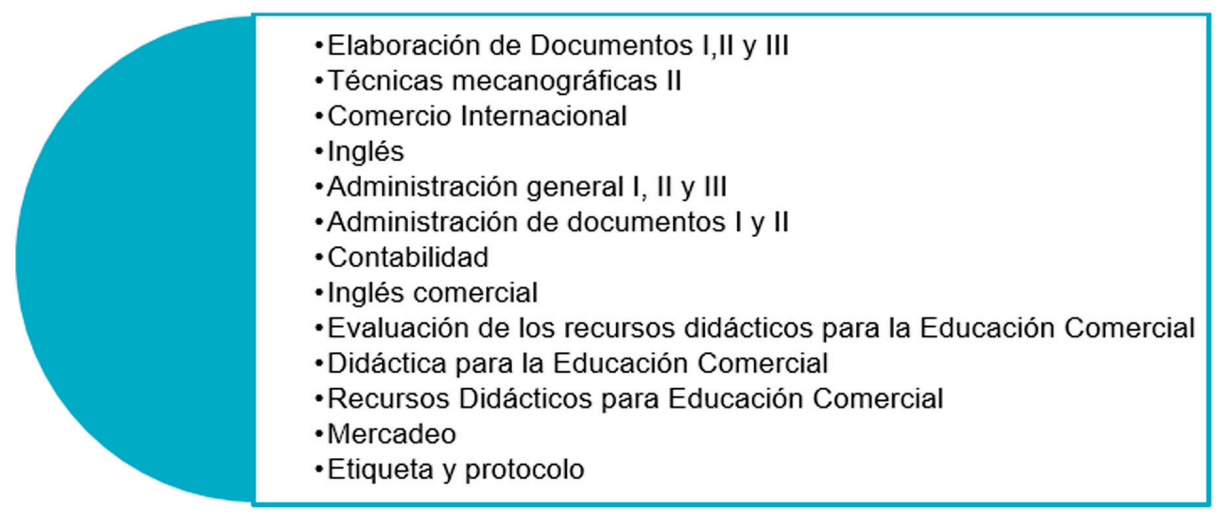

Figura 6. Cursos que el estudiantado consideró más innovadores. Fuente: elaboración propia, 2013

Aunado a la pregunta arriba expuesta, el grupo de estudiantes menciona las cualidades más importantes que debe tener un proceso de formación para que resulte innovador. Como resultado del análisis de esos datos emergieron las siguientes cualidades:

Para finalizar esta subcategoría y según muestran las respuestas de las personas encuestadas, el proceso educativo debe ser creativo e innovador, donde se desarrollen las competencias necesarias para enfrentarse a la vida como personas y profesionales. Dentro de ese contexto el modelo pedagógico de la UNA menciona que el aprendizaje implica un proceso de construcción y reconstrucción en el que las aportaciones de cada estudiante juegan un papel decisivo y le atribuyen sentido a lo que aprende en relación con su realidad. Por consiguiente es el resultado de un proceso dinámico, individual y social, donde se construyen conocimientos, se desarrollan valores, actitudes, aptitudes y habilidades, se acomodan y reorganizan nuevos esquemas de conocimiento que le permiten al estudiante comprender, reconstruir y enfrentar la realidad, así como desarrollar sus potencialidades (UNA Gaceta, 2009). Sin embargo, para que esto suceda la UNA debe asegurar una debida actualización y formación profesional a los académicos ofreciéndoles oportunidades de calidad para continuar y profundizar en su desarrollo profesional. Además, docentes y estudiantado deben romper con la idea de que 
la principal acción en el acto educativo es transmitir conocimiento. Por el contario, en la formación universitaria se debería velar por el desarrollo de transformaciones metodológicas en los cursos con el fin de generar nuevos pensamientos acorde con las demandas actuales.

\section{Competencias digitales}

Para efectos de este estudio se consideró que las competencias digitales son el conjunto de conocimientos, destrezas, habilidades y valores que deben desarrollar las personas para el uso constructivo, creativo, crítico y seguro de las Tecnologías de la Información y Comunicación (TIC) Corrales y Arce (2009). Dentro de este tema, se les consultó al grupo de estudiantes encuestado ¿cuáles competencias digitales debería poseer el estudiantado al finalizar su carrera? Las respuestas fueron diversas y las competencias mejor valoradas se resumen en la siguiente clasificación de competencias digitales:

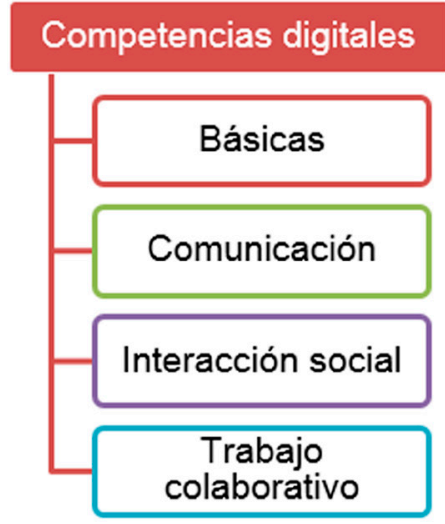

Figura 8. Clasificación competencias digitales básicas

\section{Básicas:}

- Conocer el funcionamiento básico del hardware y del software.

- Usar los sistemas operativos apropiadamente en la administración de archivos en el computador.

- Desarrollar destrezas en la digitación de textos. 
- Utilizar herramientas de productividad como procesadores de palabras y hojas electrónicas para administrar y gestionar documentos.

- Realizar búsquedas efectivas en internet de forma completa, discriminando información por medio de directorios y motores de búsquedas o metabuscadores.

- Diseñar, crear y usar bases de datos para la administración de información relacionada.

- Elaborar documentos multimedia como presentaciones y videos.

- Utilizar herramientas ofimáticas en la nube para llevar la gestión documental.

\section{Comunicación:}

- Usar frecuentemente el correo electrónico para comunicarse con otras personas.

- Usar adecuadamente herramientas tecnológicas tales como videoconferencias, foro, Facebook y blogs para intercambiar información y construir conocimiento.

- Elaborar contenidos y publicarlos en sitios web.

- Producir documentos, mensajes, ideas, preguntas y comentarios utilizando diversas herramientas tecnológicas en la nube (cloud computing).

\section{Interacción social:}

- Aprovechar de forma creativa los recursos tecnológicos para comunicarse respetuosamente con otras personas.

- Participar proactivamente en espacios virtuales de aprendizaje y redes sociales con el propósito de interactuar y crear comunidades de aprendizaje.

\section{Trabajo colaborativo:}

- Construir conocimientos en colaboración con personas.

- Aprovechar las diferentes herramientas tecnológicas para comunicarse, dialogar, producir, tomar decisiones y llegar acuerdos con otras personas. 
- Elaborar documentos digitales en colaboración con otras personas.

Figura 9. Descripción competencias digitales básicas.

La síntesis de las competencias digitales expuestas por el grupo de personas encuestadas define aquellas que debe poseer el estudiantado al finalizar la carrera de Administración de Oficinas o Educación Comercial. Sin embargo, faltan otras competencias a nivel profesional y personal relacionadas con la web 2.0 , entornos digitales y cultura digital que se deben promover en los cursos universitarios para que los estudiantes egresados de la Escuela de Secretariado Profesional sean capaces de utilizar las TIC para producir, presentar y comprender información simple y compleja y tener la habilidad necesaria para acceder a servicios y trabajar con recursos basados en Internet con una actitud crítica, colaborativa e innovadora. Por otra parte, al conocer las competencias digitales expuestas se impone la necesidad de que la Escuela de Secretariado Profesional asuma el desarrollo de procesos de innovación curricular para transformar los planes de estudio actuales. De esta forma, se hace necesario elaborar nuevos perfiles profesionales congruentes con la realidad social costarricense y latinoamericana que se transforma y avanza con el desarrollo científico y tecnológico.

\section{Discusión final}

Los resultados obtenidos, a lo largo de este estudio exploratorio, en el curso Elaboración Integral de Documentos III de las carreras Administración de Oficinas y Educación Comercial impartido en la Universidad Nacional de Costa Rica muestran con claridad que la mayoría de estudiantes encuestados tienen acceso tanto a una computadora, como a la conexión a Internet y utilizan diferentes programas para navegar y acceder a los servicios y medios en la Web. De ahí la importancia que los procesos educativos que se promueven en la universidad no pueden quedar al margen de esta realidad tecnológica y social, personal docente y estudiantado deben integrar las TIC a los cursos universitarios con nuevas situaciones comunicativas, pedagógicas y sociales por medio de proyectos innovadores apoyados 
por las TIC y con sentido para la vida del estudiantado universitario.

Los estudiantes encuestados manifiestan su opinión con respecto a los procesos de formación profesional vividos y afirman que se deben generar cambios en el rol del docente, quien debe ser especialista en la materia, pero actualizado con base en el mercado laboral, flexible y creativo para evitar pensamientos conservacionistas, además, debe ser generador de una acción pedagógica innovadora y no olvidar la siguiente función en el área afectiva: orientador y paciente con el grupo de estudiantes. Este resultado denota la importancia que las personas encuestadas han dado al proceso de formación universitaria, en donde plantean la necesidad de cambios en el rol del personal docente, contenidos, metodología y evaluación de los procesos de aprendizaje, de tal manera que la formación y actualización profesional es de suma importancia, así como los recursos necesarios para promover procesos de aprendizaje diferentes y las posibilidades de asesoramiento externo y especializado en el objeto de estudio de la Escuela de Secretariado Profesional. Ante este contexto, es relevante que el estudiantado, el personal académico y administrativo se involucren en procesos de innovación educativa acorde con sus necesidades y de esta forma transformar los planes de estudio actuales.

El estudio realizado evidencia la necesidad de seguir profundizando en el análisis de los procesos de formación universitaria y su relación con el desarrollo de competencias digitales en el estudiantado, ya que en los resultados obtenidos el estudiantado expresa algunas competencias digitales que debería poseer al finalizar la carrera de Administración de Oficinas o Educación Comercial. Sin embargo, faltan otras competencias digitales a nivel profesional y personal relacionadas con la web 2.0, entornos digitales y cultura digital que las personas encuestadas no manifestaron. Lo anterior, revela que la formación universitaria que reciben los estudiantes de las carreras de Administración de Oficinas y Educación Comercial debe analizarse a profundidad porque en este primer diagnóstico se evidencia que la formación universitaria no está respondiendo con el desarrollo integral de las competencias digitales que requiere la sociedad del conocimiento, se encuentra en un nivel inicial. De ahí la importancia de realizar una investigación más amplia, involucrando a todo el estudiantado matriculado y al grupo de académicos que conforman la Unidad Académica.

Carolina España Chavarría, Xinia Corrales Escalante 
Debido a lo anterior, es necesario que Escuela de Secretariado Profesional analice y discuta acerca de la posibilidad de realizar modificaciones a los planes de estudio y se actualice a partir de enfoques educativos centrados en el aprendiente, por ejemplo, modelo basado en competencias, y se establezcan aspectos que permitan al estudiante conocer e integrar las TIC a su profesión. En este proceso un factor determinante para el cambio son los docentes y a partir de este estudio surge la inquietud ¿cuáles son las competencias que debe desarrollar el personal académico de la UNA para conducir a la construcción del conocimiento y las competencias digitales en el estudiantado? Acerca de este tema la UNESCO en el año 2008 generó un documento titulado: "Estándares de competencias TIC para docentes". Concretamente, dicho documento expresa que para vivir, aprender y trabajar con éxito en una sociedad cada vez más compleja, rica en información y basada en el conocimiento, los estudiantes y los docentes deben utilizar la tecnología digital con eficacia. Asimismo este documento indica que en un contexto educativo sólido, las Tecnologías de la Información y la Comunicación (TIC) pueden ayudar a los estudiantes y docentes a adquirir las capacidades necesarias para llegar a ser:

- Competentes para utilizar tecnologías de la información.

- Buscadores, analizadores y evaluadores de información.

- Solucionadores de problemas y tomadores de decisiones.

- Usuarios creativos y eficaces de herramientas de productividad.

- Comunicadores, colaboradores, publicadores y productores.

- Ciudadanos informados, responsables y capaces de contribuir a la sociedad (UNESCO, 2008).

Con esta visión, somos conscientes que el personal académico de la Escuela de Secretariado Profesional debe transformarse para promover una formación universitaria innovadora y de calidad y como lo señala Santos y Guillaumín (2008) "debemos tomar conciencia del momento histórico que vive la humanidad; pensar en dónde estamos, en qué tipo de sociedad vivimos y hacia qué tipo de sociedad humana deseamos encaminarnos" (p. 81). Y a partir de ahí, emprender las formulaciones teóricas y prácticas 
en torno a la definición de la esencia de la nueva universidad en un mundo lleno de incertidumbres, paradojas y contradicciones.

Por último, cabe resaltar la urgente necesidad de que la formación inicial sea planificada y conducida de forma tal que logre contribuir al desarrollo de competencias específicas y genéricas requeridas por la sociedad. Para ello se deberá invertir en la formación docente en donde los conocimientos, las habilidades y las actitudes a desarrollar posibiliten el buen desempeño profesional y la pertinente respuesta a los problemas que enfrenta el entorno en sus múltiples escenarios.

\section{Referencias}

Area, M. (2010). ¿Por qué formar en competencias informacionales y digitales en la educación superior? Revista de Universidad y Sociedad del Conocimiento, 7(2), 2-4. Recuperado de: http:// rusc.uoc.edu/ojs/index.php/rusc/article/view/v7n2-area/v7n2area

Barrantes, R. (2013). Investigación: un camino al conocimiento, un enfoque cualitativo, cuantitativo y mixto. Costa Rica: Editorial Universidad Estatal a Distancia. Recuperado de: http://www. uned.ac.cr/editorial/resultadobusqueda.asp?codigo=U08167

Brunner, J. J. (2003). Educación e internet: ¿la próxima revolución? Chile: Fondo de Cultura Económica. Recuperado de: http:// www.fondodeculturaeconomica.com/librerias/Detalle. aspx?ctit=014376R

Castells, M. (octubre-diciembre, 2009). La apropiación de las tecnologías. Cultura juvenil en la era digital. Telos. Cuadernos de Comunicación e Innovación, (81), 111113. Recuperado de: http://telos.fundaciontelefonica.com/ docs/2010/05/11/12240001_4_4_0.pdf

Castells, M. (2005). La era de la información: Economía, Sociedad y Cultura (Vol. 1). Madrid: Alianza. 
Canales, A. y España, C. (2013). La utilidad de las TIC para la promoción de aprendizajes en la educación superior. Teoría de la Educación. Educación y Cultura en la Sociedad de la Información, 14(1),189-227. Recuperado de: http://www. redalyc.org/pdf/2010/201025739010.pdf

Corrales, X. y Arce, R. (2012). [Informe preliminar encuesta dirigida a coordinadores de ofertas educativas con una modalidad bimodal o virtual, perfil estudiante virtual]. Datos duros inéditos.

Comisión Europea. (2006). Competencias clave para el aprendizaje permanente. Un marco de Referencia Europeo. Bélgica: Comunidades Europeas. Recuperado de: http://www.mecd. gob.es/dctm/ministerio/educacion/mecu/movilidad-europa/ competenciasclave.pdf?documentld=0901e72b80685fb1

Fullan, M. (1991). The new meaning of educational change. Chicago: Teacher College Press. Recuperado de: http://store.tcpress. com/0807747653.shtml

García, C. (2003). El cambio educativo como objeto de estudio: El uso del conocimiento para la mejora de la escuela. Seminario Taller: Cultura Escolar y Cultural Profesional: Los dilemas del cambio. Costa Rica: Fundación Omar Dengo.

Goetz, J. P. y LeCompte, M. D. (1998). Etnografía y diseño cualitativo en investigación educativa. Madrid: Morata. Recuperado de: http://books.google.es/books/about/Etnograf\%C3\%ADa_y_ dise $\%$ C3\%B10_cualitativo en inv.html?id=iWpN2nsx9QgC

Hernández, R., Fernández, C. y Baptista, M. (2010). Metodología de la Investigación (5ta ed). México: McGraw-Hill/Interamericana Editores, S.A. DE C.V. Recuperado de: http://mcgraw-hill. com.mx/cgi-bin/book.pl?isbn=9701057538\&division=mexh

Instituto de Tecnologías Educativas. (2011). Competencia digital.

Recuperado de: http://recursostic.educacion.es/blogs/europa/

Carolina España Chavarría, Xinia Corrales Escalante 
media/blogs/europa/informes/Competencia_Digital_Europa ITE_marzo_2011.pdf

Santos, M. y Guillaumín, A. (2006). Avances en complejidad y educación: teoría y práctica. Barcelona: Ediciones Octaedro, S.L. Recuperado de: http://www.uv.mx/iieses/ files/2013/05/15_Avances_en_complejidad_y_educacion_2. pdf

Tedesco, J. (1995). El nuevo pacto educativo. España: Grupo Anaya.

Thomas, J. y Nelson, J. (2007). Métodos de investigación en Actividad Física. España: Editorial Paidotribo. Recuperado de: http://paidotribo.com/pdfs/844/844.i.pdf

UNESCO. (2008). Estándares de competencias en TIC para docentes. Recuperado de: http://www.oei.es/ticl UNESCOEstandaresDocentes.pdf

Universidad Nacional. (2007). Modelo pedagógico Universidad Nacional. Recuperado de: http://www.documentos.una.ac.cr/ handle/unadocs/1763 